\section{Duración y características del sangrado postparto entre las mujeres que alimentan sus bebés al seno}

Cynthia M. Visness, MA, MPH, Kathy I. Kennedy, DrPH, and Rebecca Ramos, MD

From the Contraceptive Use and Epidemiology

Division, Family Health International, Research

Triangle Park, North Carolina; and Dr. José Fabella Memorial Hospital Manila, Philippines.

\section{Obstet Gynecol 1997; 89: 159-163.}

Objetivo:Examinar la experiencia del sangrado postparto de una cohorte de mujeres que amamantan a sus bebés y compararla con la definición convencional de loquios.

Métodos: Se siguieron en forma prospectiva desde el parto a 477 mujeres con experiencia que amamantaban a sus bebés, en Manila, Filipinas, y se registró el sangrado vaginal en un diario menstrual. La duración promedio de los loquios se calculó usando un análisis por tabla de sobrevida. Además se anotó todo sangrado separado de los loquios dentro de las primeras 8 semanas postparto.

Resultados: La duración promedio de los loquios fue 27 días y no varió con la edad, paridad, sexo o peso del bebé, la frecuencia de la alimentación al seno o el nivel de suplementación. Mas de un cuarto de las mujeres experimentaron un episodio de sangrado separado de los loquios originales por al menos 4 días libres de sangrado y que empezó a mas tardar en el día 56 postparto. Diez mujeres que amamantaban pudieron tener su primer sangrado menstrual antes del día 56.

Conclusiones: Los loquios duraron substancialmente mas tiempo que la presunción convencional de 2 semanas. Fue común que el sangrado postparto se detenga y se inicie de nuevo o que se caracterice por manchado o sangrado intermitente. El retorno de las menstruaciones es raro entre las mujeres que brindan alimentación al seno exclusiva en las primeras 8 semanas postparto.

\section{Fertilidad de las mujeres que practican la lactan- cia exclusiva en el período postparto temprano}

Cynthia M. Visness, MPH, Kathy I. Kennedy, DrPH, Barbara A. Gross, PhD, Suzanne Parenteau-Carreau, MD, Anna M. Flynn, MD, and James B. Brown, PhD

From the contraceptive Use and Epidemiology Division, Family Health International, Research Triangle Park, North Carolina; the Department of Clinical

Endocrinology, Westmead Hospital, Westmead, NSW, Australia; SERENA CANADA, Montreal, Canada; the Department of Obstetrics and Gynecology, Birmingham Maternity Hospital, Birmingham England; and the Department of Obstetrics and Gynecology, University of Melbourne, Melbourne, VIC, Australia.

\section{Obstet Gynecol 1997; 89: 164-167.}

Objetivo: Examinar la hemorragia entre las 6 y 8 semanas postparto en mujeres que ofrecen lactancia ma- terna exclusiva y su asociación con la fertilidad determinada por análisis hormonal.

Métodos: Se siguieron setenta y dos mujeres con lactancia exclusiva en forma prospectiva desde los 42 días postparto. El sangrado vaginal se registró diariamente. Se compararon las mujeres que presentaron sangrado se compararon con aquellas que no lo presentaron con respecto al tiempo de ovulación y el tiempo de la primera menstruación.

Resultados: casi la mitad de las mujeres presentaron algún tipo de sangrado o manchado entre las 6 y 8 semanas postparto. Estas mujeres eventualmente menstruaron y ovularon mas tempranamente que aquellas que no presentaron sangrado, pero las diferencias no fueron significativas. El estudio tenía un poder del 34 y $45 \%$ para detectar una diferencia del $20 \%$ en la proporción de mujeres ovulando y menstruando, respectivamente, a los 6 meses postparto, y un poder de 10 y $16 \%$ para detectar las mismas diferencias al año. Siete mujeres experimentaron desarrollo folicular ovárico antes del día 56, pero ni la hemorragia ni el desarrollo folicular se asociaron con ovulación en ninguna mujer en las primeras 8 semanas postparto.

Conclusiones: Es improbable que la hemorragia vaginal postparto en mujeres que practican lactancia exclusiva en las primeras 8 semanas postparto represente un retorno a la fertilidad.

\section{Resultado obstétrico en embarazo prolongado no complicado}

Z.M. Abotalib, M.H. Soltan, N. Chowdhury, B. Adelusi

Department of Obstetrics \& Gynaecology, King Khalid University, College of Medicine, King Saud University, Riyadh, Saudi Arabia

\section{International Journal of Gynecology \& Obstetrics 1996; 55: 225-230.}

Objetivo: Estudiar y comparar el resultado obstétrico en embarazos a término y embarazos prolongados no complicados.

Métodos: El estudio fue una revisión retrospectiva de 596 casos. Se analizaron las historias de 286 pacientes con embarazos prolongados no complicados ( $\geq 42$ semanas) y 310 pacientes con embarazos normales a término $(37$ a $<42$ semanas) se usó el paquete Gold Stat para la codificación y análisis estadísticos.

Resultados: No hubo morbilidad ni mortalidad fetal entre los dos grupos. Sin embargo el número de abortos previos fue significativamente mayor en los embarazos prolongados comparados con los embarazos a término. En forma similar, los pesos fetal y placentario, tasas de inducción del trabajo de parto y tasas de parto operatorio fueron significativamente mayores en los embarazos prolongados. Sin embargo, no hubo diferencias significativas entre las pacientes quienes fueron inducidas y aquellas que tuvieron parto espontáneo entre los embarazos prolongados. 
Conclusiones: No hay duda de que el embarazo prolongado se puede asociar con ciertos factores de riesgo. Sin embargo, no hay variable de resultado para justificar la inducción o no de aquellos embarazos que son prolongados.

\section{Eritropoyetina, factor estimulante de granulocitos-colonias, interleukina $13 \mathrm{E}$ interleukina 6 durante el ciclo menstrual normal}

S. Makidona, M. Mikuni, M. Sogame, Y. Kobamatsu, I. Furuta, H. Yamada, R. Yamamoto, S. Fujimoto

Department of Obstetrics and Gynecology, Hokkaido University School of Medicine, Sapporo, Japan

\section{International Journal of Gynecology \& Obstetrics 1996; 55: 265-271.}

Objetivo: La menstruación y la ovulación son los principales eventos durante el ciclo menstrual. La menstruación puede influir el recuento sanguíneo completo debido a la pérdida de sangre. Puesto que la ovulación es un fenómeno similar al inflamatorio, los recuentos de leucocitos pueden cambiar durante la ovulación. Por estas razones, se investigó el recuento sanguíneo completo y las concentraciones séricas periféricas de citokinas asociadas durante el ciclo menstrual normal.

Materiales y métodos: Mujeres voluntarias normales ( $n=9$; edad 21-33 años), con ciclos menstruales de 25-33 dias registraron la temperatura basal cada día y se les tomó muestra de sangre venosa tres veces por semana. Se hizo un recuento sanguíneo completo y se midieron la eritropoyetina, factor estimulante de granulocitos-colonias, interleukina $1 \beta$, interleukina 6 y hormonas $(\mathrm{FSH}$, $\mathrm{LH}$, estradiol y progesterona).

Resultados: Los eritrocitos, leucocitos totales, recuentos de granulocitos y de plaquetas no mostraron cambios estadísticamente significativos durante el ciclo menstrual utilizando el análisis de varianza con el test $\mathrm{F}$ de Scheffe. No se observaron cambios en la eritropoyetina, interleukina $1 \beta$, e interleukina 6 a lo largo del ciclo menstrual. Por contraste, las concentraciones del factor estimulante de granulocitoscolonias aumentaron significativamente a $28.0 \pm 3.1 \mathrm{pg} / \mathrm{ml}$ (promedio $\pm \mathrm{SEM}$ ) durante la fase ovulatoria, comparada con $10.7 \pm 0.6 \mathrm{pg} / \mathrm{ml}$ en otras fases.

Conclusión: Estos resultados indican que la pérdida sanguínea menstrual no afecta el recuento sanguíneo completo y sugiere que el factor estimulante de granulocitos-colonias juega un importante papel en el mecanismo de ovulación.

\section{Extensión de la duración de las píldoras anticonceptivas orales para manejar los síntomas de supresión hormonal}

Patricia J. Sulak, MD, Brian E. Cressman, MD, Enid Waldrop, RNC, Sonia Holleman, AAS, and Thomas J. Kuehl, PhD

From the Departments of Obstetrics and Gynecology, Pathology, and Medical Biochemistry and Genetics, Scott \& White Clinic and Memorial Hospital, Texas A\&M University Health Science Center College of Medicine, Temple, Texas

Obstet Gynecol 1997; 89: 179-183.
Objetivo: Probar la hipótesis que extendiendo el número de contraceptivos orales activos (CO) admnistrados, podría disminuirse la frecuencia de problemas relacionados con la menstruación incluyendo dismenorrea, menorragia, síntomas de tipo premenstrual y migrañas menstruales.

Métodos: Se diseñó un análisis prospectivo para seguir las experiencias de 50 mujeres que tomaban $\mathrm{CO}$ y presentaban problemas relacionados con la menstruación. Se siguieron 50 mujeres consecutivas, quienes estaban tomando $\mathrm{CO}$ y tenían síntomas durante el intervalo libre de píldoras, en una clínica de multiespecialidades por un equipo de médico y enfermera. Se les permitió a las pacientes extender el número de $\mathrm{CO}$ activos consecutivos para retardar los síntomas relacionados con la menstruación.

Resultados: El resultado inmediato de las 50 pacientes reveló que el $74 \%$ ( 37 pacientes) se estabilizaron en un régimen extendido de 6 a 12 semanas de días consecutivos con CO activos. Veintiseis por ciento (13 pacientes) descontinuaron los $\mathrm{CO}$ o regresaron al régimen estándar con 3 semanas de píldoras activas. De las 37 pacientes que se estabilizaron en un régimen extendido, 27 han completado entre 5 y 13 ciclos extendidos con 6-23 meses de seguimiento (promedio 16 meses).

Conclusión: La experiencia con una serie de 50 usuarias de $\mathrm{CO}$ con síntomas relacionados con la menstruación demostró que retardar la menstruación extendiendo el número de días consecutivos de píldoras activas es bien tolerado y eficaz. Se amerita un gran estudio prospectivo para aumentar el conocimiento en ésta área.

\section{Determinantes etiológicas del abruptio placentae}

Michael S. Kramer, MD, Robert H. Usher, MD, Raphael Pollack, MD, Mark Boyd, MD, and Susan Usher, MA

From the departments of Epidemiology and Biostatistics, Pediatrics, and Obstetrics and Gynecology, McGill University Faculty of Medicine, Montreal, Quebec, Canada

$$
\text { Obstet Gynecol 1997; 89: 221-226. }
$$

Objetivo: Cuantificar los papeles de los determinantes sospechados de tipo sociodemográfico, antropométricos, de comportamiento y patológicos en la etiología del abruptio placentae.

Métodos: Se hizo un estudio de cohorte hospitalaria de 36,875 partos no referidos entre enero de 1978 y marzo de 1989. La edad gestacional se basó en las fechas menstruales confirmada (dentro de 7 días) por ultrasonido temprano.

Resultados: La paridad, educación materna, peso antes de la gestación y la tasa de ganancia neta de peso en la gestación no tienen asociaciones independientes significativas con el abruptio. Las determinantes significativas incluyeron las siguientes: parto de recién nacidos muy pequeños para la edad gestacional (OR 3.99; IC 95\% $2.75,5.77$ ). Corioamnionitis (OR 2.5; IC $95 \% 1.58,3.98$ ), ruptura prolongada de membranas (OR 2.38; IC 95\% $1.55,3.65$ ), pre-eclampsia (OR 2.05; CI 95\% 1.39,304), hipertensión asociada a la gestación sin albuminuria (OR 1.57 , IC $95 \% 1.00,2.46)$, hipertensión pre-gestacional 